\title{
ASPECTOS BIOLÓGICOS DE Trichogramma galloi Zucchi, 1988 (Hymenoptera: Trichogrammatidae) CRIADOS EM OVOS DE Diatraea saccharalis (Fabricius, 1794) (Lepidoptera: Crambidae)
}

\author{
Biological aspects of Trichogramma galloi Zucchi, 1988 (Hymenoptera: Trichogrammatidae) \\ reared on eggs of Diatraea saccharalis (Fabricius, 1794) (Lepidoptera: Crambidae)
}

\author{
Josean Leite Pereira-Barros ${ }^{1}$, Sônia Maria Forti Broglio-Micheletti ${ }^{2}$, Adriano Jorge Nunes dos Santos ${ }^{3}$, \\ Lucyo Wagner Torres de Carvalho ${ }^{3}$, Luiz Henrique Torres de Carvalho ${ }^{3}$, Carlos José Tavares de Oliveira ${ }^{3}$
}

\section{RESUMO}

Alguns aspectos biológicos de Trichogramma galloi Zucchi, 1988 (Hymenoptera: Trichogrammatidae) criados em ovos de Diatraea saccharalis (Fabricius, 1794) (Lepidoptera: Crambidae), foram estudados em laboratório a $26 \pm 2{ }^{\circ} \mathrm{C}$; UR $60 \pm 10 \%$ e fotofase de 12 horas. Os resultados obtidos mostraram um período médio de desenvolvimento pré-imaginal de 9,46 $\pm 0,7$ dias e uma viabilidade média de 78,05\%. O número médio de $T$. galloi emergido por ovo do hospedeiro foi de 2,29. Nas criações em laboratório, as fêmeas de T. galloi foram predominantes. A longevidade de machos e fêmeas foi, em média 3,26 $\pm 0,12$ dias (sem alimento) e $6,36 \pm 0,19$ dias (alimentando-se com mel). Os ovos de D. saccharalis de primeiro dia apresentaram parasitismo médio de 79,3 $\pm 5,25 \%$ e os de segundo dia $55,2 \pm 2,33 \%$.

Termos para indexação: Biologia, controle biológico, parasitóide.

\begin{abstract}
Some biological aspects of the parasitoid Trichogramma galloi Zucchi, 1988 (Hymenoptera: Trichogrammatidae) reared on eggs of Diatraea saccharalis (Fabricius, 1794) (Lepidoptera: Crambidae) were studied in the laboratory, at $26^{\circ} \mathrm{C}, \mathrm{UR} 60 \pm 10 \%$ and 12 hours photophase. The studies showed a life cycle, from egg to adult, of $9.46 \pm 0.7$ days and a survival rate of $78.05 \%$. The mean number of $T$. galloi emerged by egg of sugar cane borer was of 2.29. Females were predominant in the laboratory rearing and the longevity of males and females was, on the average, $3.26 \pm 0.12$ days (without feeding) and $6.36 \pm 0.19$ days (feeding with honey). The parasitism was between $79.3 \pm 5.25 \%$ and $55.2 \pm 2.33 \%$, in eggs of first and second days, respectively.
\end{abstract}

Index terms: Biology, biological control, parasitoid.

\section{(Recebido para publicação em 6 de abril de 2004 e aprovado em 23 de junho de 2005)}

\section{INTRODUÇÃO}

A cultura da cana-de-açúcar é de grande importância econômica para alguns países das Américas, especialmente para o Brasil que se destaca como um dos principais produtores mundiais de açúcar e álcool de cana. No Nordeste, a cultura canavieira compreende a Zona da Mata dos Estados da Paraíba, Pernambuco, Alagoas e Sergipe até o recôncavo baiano. No Estado de Alagoas, a produção de cana-de-açúcar atinge uma área de 450 mil hectares, favorecida pelas condições agroclimáticas (MENDONÇA, 1996).

Apesar da facilidade de adaptação ao clima do Brasil, a cultura enfrenta uma série de problemas fitossanitários, tais como a incidência de insetos-praga, como as brocas do gênero Diatraea (Lepidoptera), que fazem diminuir a produtividade, acarretando em prejuízo econômico para os produtores (BOIÇA JÚNIOR et al., 1997).

Atualmente, o controle mais eficiente de Diatraea spp. tem sido o biológico empregando o endoparasitóide larval Cotesia flavipes (Cameron, 1891) (Hymenoptera: Braconidae). Entretanto, o fator chave de crescimento da população das brocas-da-cana é a fase de ovo (BOTELHO, 1985), que apresenta abundância de espécies de parasitóides, especialmente as do gênero Trichogramma (Hymenoptera) (BOTELHO et al., 1999; LOPES, 1988; SALES JÚNIOR \& PARRA, 1993; ZUCCHI, 1985).

Algumas espécies de Trichogramma vêm sendo utilizadas em liberações inundativas em diversos países, para o controle de pragas de significância agrícola (SMITH, 1996). Embora tenham preferência por ovos de Lepidoptera, parasitam também, cerca de 200 espécies pertencentes a mais de 70 famílias de Insecta e concernentes a oito ordens de importância agrícola (MORRISON, 1985).

Os fatores climáticos são as causas direta e indireta das flutuações e da dinâmica populacional da broca. Eles modificam a duração do ciclo biológico e sua capacidade

\footnotetext{
${ }^{1}$ Mestre em Agronomia (Área de Concentração: Produção Vegetal) - Centro de Ciências Agrárias/CECA/UFAL - Rio Largo, AL - leitepb@uol.com.br

${ }^{2}$ Professor Adjunto, Departamento de Fitotecnia e Fitossanidade - CECA/UFAL - Rio Largo, AL - 57100-000 - vlamiche@ofm.com.br

${ }^{3}$ Estudantes do Curso de Agronomia do CECA/UFAL - Rio Largo, AL - Bolsistas de Iniciação Científica do CNPq e da Fundação de Amparo à Pesquisa em Alagoas (FAPEAL).
} 
reprodutiva e, principalmente, exercem pressões sobre seus diversos inimigos naturais criando assim desequilíbrios nas inter-relações entre o organismo fitófago e o hospedeiro (TERÁN, 1982). Entre os fatores físicos, a temperatura é o de maior influência sobre a fecundidade, duração do ciclo de desenvolvimento, razão sexual, viabilidade e longevidade dos parasitóides (HARRINSON et al., 1985; NOLDUS, 1989). Cônsoli \& Parra (1996) constataram que o ciclo biológico de duas espécies de Trichogramma criadas em ovos de Diatraea saccharalis (Fabricius, 1794) (Lepidoptera: Crambidae) e Anagasta kuehniella (Zeller, 1879) (Lepidoptera: Pyralidae), pode ser afetado por diversos fatores. Lopes (1988) constatou que o parasitismo por Trichogramma galloi Zucchi, 1988 (Hymenoptera: Trichogrammatidae) foi maior quando mantido constantemente em ovos de D. saccharalis do que quando mantido em ovos de A. kuehniella.

Objetivou-se com este trabalho avaliar a taxa de parasitismo, viabilidade, razão sexual, número de parasitóides por ovo, desenvolvimento pré-imaginal e longevidade de machos e fêmeas de $T$. galloi, multiplicados em ovos de $D$. saccharalis, visando sua criação massal e posterior liberação em campo.

\section{MATERIALEMÉTODOS}

O experimento foi realizado no Laboratório de Entomologia, do Centro de Ciências Agrárias da Universidade Federal de Alagoas, Rio Largo, AL, a $26 \pm 2{ }^{\circ} \mathrm{C}$, UR de $60 \pm 10 \%$ e fotofase de 12 horas.

A espécie $T$. galloi foi coletada em canaviais pertencentes à Usina Triunfo Agro-Industrial S.A., situada no município de Boca da Mata, Alagoas, parasitando ovos de Diatraea spp. A identificação foi realizada pelo Prof. Dr. Roberto Antonio Zucchi, da ESALQ-USP.

Os parasitóides foram multiplicados em ovos de $D$. saccharalis e mantidos em recipientes de vidro de $17 \mathrm{~cm} \mathrm{x}$ $10,5 \mathrm{~cm}$, vedados por filme de PVC laminado perfurado com alfinete entomológico, para aeração. Para a alimentação dos adultos de T. galloi, uma gotícula de mel foi depositada na superfície interna dos recipientes de criação. Indivíduos oriundos dessa criação foram utilizados nos experimentos.

Parasitismo, desenvolvimento pré-imaginal, viabilidade dos ovos de $D$. saccharalis parasitados e número de $T$. galloi emergido - Posturas de D. saccharalis com 24 e 48 horas ( 100 ovos em cada idade) foram oferecidas a $T$. galloi durante 48 horas, usando-se a proporção de cinco adultos do parasitóide/ovo da praga, e individualizadas em tubos de ensaio $(10 \mathrm{~cm} \mathrm{x} 1,5 \mathrm{~cm})$ vedados por PVC laminado. Após sete dias, avaliou-se a porcentagem de parasitismo, por meio da contagem dos ovos escurecidos em relação ao número total de ovos. $\mathrm{O}$ delineamento experimental foi inteiramente casualizado, com 100 repetições e as médias dos tratamentos comparadas pelo teste F, a $1 \%$ de probabilidade. Em cada tubo, foi avaliado o período entre o oferecimento dos ovos aos parasitóides e a emergência dos adultos, o que caracterizou o período de desenvolvimento pré-imaginal. A viabilidade dos ovos de $D$. saccharalis foi avaliada em cada postura, observando-se o número de ovos com orifício de emergência e o número de ovos parasitados, estabelecendo-se a razão entre esses valores. A razão entre o número total de parasitóides por massa de ovos e o número de ovos parasitados com orifício de emergência representou o número de adultos por ovo de $D$. saccharalis.

Longevidade de $T$. galloi com e sem alimento - Para avaliação da longevidade com alimento, colocou-se uma gotícula de mel puro na superfície interna do tubo de ensaio quando os parasitóides iniciaram a emergência. Diariamente, fizeram-se duas observações, com intervalo de 12 horas, desde a emergência do primeiro até a morte do último parasitóide. O delineamento experimental foi inteiramente casualizado, com 50 repetições e as médias dos tratamentos comparadas pelo teste $\mathrm{F}$ a $5 \%$ de probabilidade.

Razão sexual de T. galloi - Para o conhecimento da razão sexual foram separadas 14 amostras de adultos de T. galloi, cada uma delas contendo 100 exemplares multiplicados em laboratório. O número total da amostra foi de 489 machos e 911 fêmeas de $T$. galloi. A separação por sexo foi realizada por um estereomicroscópio com aumento de $80 \mathrm{X}$, baseando-se nas características morfológicas das antenas (BOWEN \& STERN, 1966). A proporção sexual encontrada foi analisada pelo teste $\mathrm{X}^{2}$.

\section{RESULTADOS E DISCUSSÃO}

Os ovos de D. saccharalis de primeiro dia apresentaram parasitismo médio de $79,3 \pm 5,2 \%$ e os de segundo dia 55,2 $\pm 2,3 \%$. Houve diferença significativa na porcentagem de parasitismo, quando se considerou a idade dos ovos, evidenciando que o parasitóide tem a preferência por ovos mais novos. Segundo Mellini (1986), a idade do hospedeiro pode restringir a aceitação pelo parasitóide, em função do endurecimento do córion, dificultando a 
penetração do ovipositor. Portanto, na multiplicação dos parasitóides em laboratório, a maior taxa de parasitismo possivelmente ocorrerá para ovos com até 24 horas.

A duração média do período ovo-adulto para $T$. galloi criado em ovos de D. saccharalis foi de 9,46 \pm 0,7 dias. Este resultado foi semelhante ao obtido por Alencar et al. (2000) que verificaram uma duração média de 9,42 dias para Trichogramma pretiosum Riley, 1879 criado em ovos de Sitotroga cerealella (Olivier, 1819) (Lepidoptera: Gelechiidae) mantidos a $250,9^{\circ} \mathrm{C}$. Stein \& Parra (1987) também observaram uma duração média de 9,9 dias para esse mesmo parasitóide criado em ovos de Phthorimaea operculella (Zeller, 1873) (Lepidoptera: Gelechiidae) a uma temperatura de $25^{\circ} \mathrm{C}$.

A viabilidade dos ovos parasitados foi de $78,1 \%$, semelhante aos resultados obtidos por Oliveira et al. (2003), que encontraram uma viabilidade entre 77 e $79 \%$ em ovos de Oxydia vesulia (Cramer, 1779) (Lepidoptera: Geometridae) com três e cinco dias de idade. No entanto, Alencar et al. (2000) observaram viabilidade de $88 \%$ em ovos de $S$. cerealella parasitados por T. pretiosum, quando estudaram a biologia desse parasitóide.

O número médio de adultos de $T$. galloi emergidos por ovo de $D$. saccharalis foi de 2,29 $\pm 0,43$. Resultados semelhantes foram encontrados por Oliveira et al. (2003), que constataram de 2 a 3 parasitóides/ovo, utilizando como hospedeiros ovos de $O$. vesulia e de $D$. saccharalis. O número de espécimes de Trichogramma sp. que se desenvolve por ovo do hospedeiro é variável, sendo maior em ovos de maior tamanho, fato constatado por Parra \& Zucchi (1986).

A razão sexual de $T$. galloi foi 0,64 (1,55 fêmeas para 1,0 macho) a $26 \pm 2^{\circ} \mathrm{C}$. Essa relação poderá variar em função de fatores do ambiente (SUZUCHI, 1982; VOEGELÉ, 1978), densidade e tipo do hospedeiro (SUZUCHI et al., 1984). Quando se deseja fazer o controle biológico é importante que o número de fêmeas seja maior do que o de machos, pois cabe a elas, a localização e o parasitismo do hospedeiro (GARCIA, 1991).

A longevidade para machos e fêmeas foi em média 3,26 $\pm 0,12$ dias para insetos sem alimento e 6,36 $\pm 0,19$ dias para aqueles que foram alimentados. Stein \& Parra (1987) encontraram uma longevidade entre 6 e 7 dias, para Trichogramma sp. criado nos hospedeiros A. kuehniella, S. cerealella e Plodia interpunctella (Hübner, 1813) (Lepidoptera: Pyralidae), sendo que nesse caso, os parasitóides não foram alimentados. Foi ainda relatado que ao utilizarem parasitóides alimentados, a longevidade variou entre 10 a 12 dias.

\section{CONCLUSÕES}

a) Ovos de D. saccharalis com até 24 horas permitiram maior porcentagem de parasitismo por T. galloi;

b) A emergência de $T$. galloi em ovos de $D$. saccharalis, a partir do parasitismo, ocorre em até 10 dias;

c) A viabilidade dos ovos de $D$. saccharalis parasitados por T. galloi, próxima a $80 \%$, garante êxito na criação massal;

d) A liberação de T. galloi para o controle de $D$. saccharalis deve ser realizada com insetos recémemergidos, em virtude desse parasitóide apresentar curta longevidade;

e) Cada ovo de D. saccharalis parasitado origina dois adultos do inimigo natural, o que possibilita a quantificação de T. galloi a ser liberado;

f) Nas criações em laboratório, as fêmeas de $T$. galloi são predominantes.

\section{AGRADECIMENTOS}

Ao Banco do Nordeste pelo financiamento da pesquisa; à Usina Triunfo Agroindustrial S.A. por ceder adultos de $D$. saccharalis para a criação de $T$. galloi em laboratório; ao Prof. Dr. Roberto Antonio Zucchi da ESALQ/USP pela identificação de T. galloi e à FAPEAL (Fundação de Amparo à Pesquisa em Alagoas) pelo apoio na concessão de bolsas de Iniciação Científica e de Mestrado.

\section{REFERÊNCIASBIBLIOGRÁFICAS}

ALENCAR, J.A.; HAJI, F. N.P.; OLIVEIRA, J. V.; MOREIRA, A. N. Biologia de Trichogramma pretiosum Riley em ovos de Sitotroga cerealella (Olivier). Pesquisa Agropecuária Brasileira, Brasília, v. 35, n. 8, p. 1669-1674, 2000.

BOIÇA JÚNIOR, A. L.; LARA, F. M.; BELLODI, M. P. Influència de variedades de cana-de-açúcar, incorporadas em dieta artificial, no desenvolvimento de Diatraea saccharalis (Fabr.) e no seu parasitismo por Cotesia flavipes (Cam.). Anais da Sociedade Entomológica do Brasil, Londrina, v. 26, n. 3, p. 537-542, 1997.

BOTELHO, P. S. M. Tabela de vida ecológica e simulação da fase larval da Diatraea saccharalis (Fabr., 1794) (Lep., Pyralidae). 1985. 110 f. Tese (Doutorado) - Escola Superior de Agricultura "Luiz de Queiroz", Universidade de São Paulo. Piracicaba, 1985. 
BOTELHO, P. S. M.; PARRA, J. R. P.; CHAGAS NETO, J. F.; OLIVEIRA, C. P. B. Associação do parasitóide de ovos Trichogramma galloi Zucchi (Hymenoptera: Trichogrammatidae) e do parasitóide larval Cotesia flavipes (Cam.) (Hymenoptera: Braconidae) no controle de Diatraea saccharalis (Fabr.) (Lepidoptera: Crambidae) em cana-de-açúcar. Anais da Sociedade Entomológica do Brasil, Londrina, v. 28, n. 3, p. 491496, 1999.

BOWEN, W. R.; STERN, V. M. Effect of temperature on the production of males and sexual mosaics in a uniparental race of Trichogramma semifumatum (Hymenoptera: Trichogrammatidae). Annals of the Entomological Society of America, Columbus, v. 59, n. 4, p. 823-834, 1966.

CÔNSOLI, F. L.; PARRA, J. R. P. Produção "in vitro" de parasitóides: criação de Trichogramma galloi Zucchi e $T$. pretiosum Riley no Brasil. In: PARRA, J. R. P.; ZUCCHI, R. A. (Orgs.). Curso de Controle Biológico com Trichogramma. [S.1.: s.n.], 1996. p. 89-97.

GARCIA, M. A. Ecologia das interações inseto/planta. In: PANIZZI, A. R.; PARRA, J. R. P. (Eds.). Ecologia nutricional de insetos e suas implicações no manejo integrado de pragas. São Paulo: Manole/CNPq, 1991.359 p.

HARRINSON, W.; KING, E.; OUZTS, J. Development of Trichogramma exigum and Trichogramma pretiosum at five temperatures regimen. Environmental Entomology, College Park, v. 14, p. 118-121, 1985.

LOPES, J. R. S. Estudos bioetológicos de Trichogramma galloi Zucchi, 1988 (Hym., Trichogrammatidae) para o controle de Diatraea saccharalis (Fabr., 1794) (Lep., Pyralidae). 1988. 141 f. Dissertação (Mestrado em Entomologia) - Escola Superior de Agricultura "Luiz de Queiroz”, Universidade de São Paulo. Piracicaba, 1988.

MELLINI, E. Importanza dell' etá dell' uovo, al momento della parassitizzazione, per la biologia degli imenotteri oofagi. Bolletino dell' Intituto di Entomologia "Guido Grandi” della Univesitá de Bologna, Bologna, v. 41, p. 121, 1986.
MENDONÇA, A. F. (Ed.). Pragas da cana-de-açúcar. [S.l.]: Insetos \& Cia, 1996.200 p.

MORRISON, R. K. Trichogramma spp. In: SINGH, P.; MOORE, R. F. (Eds.). Handbook of insect rearing. [S.l.: s.n.], 1985. v. 1, p. 413-417.

NOLDUS, L. P. J. J. Semiochemicals, foraging behaviour and quality of entomophagous insects for biological control. Journal of Applied Entomology, Hamburg, v. 108, p. 425-451, 1989.

OLIVEIRA, H. N.; PARATISSOLI, D.; ZANUNCIO, J. C.; SERRÃO, J. E. Influência da idade dos ovos de Oxydia vesulia no parasitismo de Trichogramma maxacali. Pesquisa Agropecuária Brasileira, Brasília, v. 38, n. 4, p. 551-554, 2003.

PARRA, J. R. P.; ZUCCHI, R. A. Uso de Trichogramma no controle de pragas. In: NAKANO, O.; SILVEIRA NETO, S.; PARRA, J. R.; ZUCCHI, R. A. (Orgs.). Atualização sobre métodos de controle de pragas. Piracicaba: FEALQ, 1986. p. 54-75.

SALES JÚNIOR, O.; PARRA, J. R. P. Preferência hospedeira de Trichogramma galloi Zucchi, 1988, criado sobre ovos de Anagasta kuehniella (Zeller, 1879) e Diatraea saccharalis (Fabr., 1794). In: CONGRESSO BRASILEIRO DE ENTOMOLOGIA, 14., 1993, Piracicaba. Resumos... Piracicaba: Sociedade Entomológica do Brasil, 1993.p. 262.

SMITH, S. M. Biological control with Trichogramma: advances, sucessess, and potential of their use. Annual Review of Entomology, Palo Alto, v. 41, n. 1, p. 375-406, 1996.

STEIN, C. P.; PARRA, J. R. P. Aspectos biológicos de Trichogramma sp. em diferentes hospedeiros. Anais da Sociedade Entomológica do Brasil, Campinas, v. 16, n. 1, p. 163-171, 1987.

SUZUCHI, Y. Sex-ratio control in Trichogramma. In: INRA. Les Trichogrammes. Antibes, 1982. p. 151. 
SUZUCHI, Y.; TSUJI, H.; SASAKAWA, M. Sex allocation and effects of superparasitism on secondary sex ratios in the gregarious parasitoid, Trichogramma chilonis (Hymenoptera: Trichogrammatidae). Animal Behavior, Bloomington, v. 32, p. 478-484, 1984.

TERÁN, F. O. Pragas da cana-de-açúcar. In: SEMINÁRIO DE TECNOLOGIAAGRONÔMICA, 1., 1982, Piracicaba, SP. Anais... Piracicaba: Copersucar, 1982. p. 117-132.
VOEGELÉ, J. Utilisation des Trichogrammes. In: INRA. Station de zoologie et de lutte biologique 14-AGRO-436. Antibes, 1978.p. 447-452.

ZUCCHI, R. A. Taxonomia de espécies de Trichogramma (Hym., Trichogrammatidae) associados a algumas pragas (Lepidoptera) no Brasil. 1985. 77 f. Tese (Livre Docência) - Escola Superior de Agricultura "Luiz de Queiroz", Universidade de São Paulo, Piracicaba, 1985. 